\title{
Subacute Onset Hand Clumsiness in a Renal Transplant Patient with a Recent ICU Stay
}

\author{
William Bradford, MD
}

\section{INTRODUCTION}

Solid organ transplant recipients carry a high burden of disease and are susceptible to opportunistic infections not seen in other patient subgroups. Signs and symptoms that would not necessarily warrant timely attention in other patient groups may call for an aggressive workup in this subset of patients. Here, we present one case in which a seemingly trivial complaint denoted serious underlying pathology, and timely attention to this minor but unusual concern led to early intervention with appropriate treatment of an uncommon disorder.

\section{CASE DESCRIPTION}

The patient is a 47-year-old female with a history of renal transplant due to lupus glomerulonephropathy on tacrolimus, prednisone, hydroxychloroquine, and mycophenolate for immunosuppression and recurrent otitis media who presented to the emergency department for evaluation of ear pain consistent with her prior episodes of otitis media. Her past medical history was otherwise notable for a recent intubation for flash pulmonary edema following hypertensive emergency at an outside hospital. Review of systems was notable for progressive bilateral hand clumsiness over the past few days as well as an area of painful swelling in the right calf. Her neurological examination was remarkable for weakness of the bilateral hand intrinsic muscles, diminished left hand proprioception, and agraphia, but was otherwise normal. She underwent computed tomography of the head without contrast, which revealed multiple areas of vasogenic edema in the white matter as well as a left parotid abscess.

\section{DIFFERENTIAL DIAGNOSIS}

The findings of agraphia and diminished proprioception on neurological examination localized the lesion to the brain-with agraphia, her most specific finding, most associated with lesions in the language-dominant superior parietal lobe. ${ }^{1}$ The differential diagnosis for CNS pathologies in the immunocompromised host, while very broad, should focus principally on infectious, vascular, and neoplastic causes. Specific considerations for this patient included intracranial hemorrhage, posterior reversible leukoencephalopathy (given recent hypertensive emergency), brain abscess, primary or secondary CNS neoplasm, and CNS vasculitis.

\section{OUTCOME AND FOLLOW UP}

Follow-up MRI brain confirmed the diagnosis of multiple brain abscesses as well as a left parotid abscess. Otolaryngology and interventional radiology were consulted for parotid abscess aspiration. Gram stain of this fluid demonstrated modified acid-fast branching gram-positive rods. Following the CT head, the patient was loaded with prophylactic antiepileptic drugs. Once the organism was identified, the patient was started on intravenous imipenem and sulfamethoxazoletrimethoprim (SMX-TMP). She was admitted to the neurological intensive care unit for close monitoring of her neurological status and monitoring for seizure activity. The area of calf swelling was later determined to be an abscess that was drained and revealed gram stain findings similar to the parotid abscess. Cultures from both areas as well as the blood eventually grew Nocardia farcinical Nocardia kroppenstedtii. She underwent TEE 3 days after admission which was negative for any vegetation. Her hospital course was lengthy and complicated by refractory multifactorial thrombocytopenia, acute DVT, and acute hypoxic respiratory failure due to flash pulmonary edema.

\section{DISCUSSION}

A new focal neurological deficit in any patient should prompt an expedient and aggressive workup. Evaluation for stroke should be considered soon after such a deficit is identified with prompt consideration for thrombolytic therapy if appropriate. In the case of this patient, her hand numbness and clumsiness represented a minor incidental complaint for the patient, but the emergency providers rightly maintained a high index of suspicion for serious underlying pathology in the setting of a new neurological deficit and opted for early head imaging. A low threshold for head imaging is appropriate in the transplant population, and likely prevented a serious adverse outcome in this case.

Nocardiosis is an uncommon gram-positive bacterial infection caused by aerobic bacteria in the genus Nocardia. Disseminated nocardiosis (DN) typically occurs in immunocompromised patients (approximately 2/3 of cases), but it can also occur in immunocompetent patients. DN can affect virtually any organ system and is typically highly treatment-resistant, requiring prolonged courses of intravenous antibiotics and multiple source control procedures. ${ }^{2}$ 
The risk of nocardiosis is highest in the first year following solid organ transplant. Heart and lung transplant recipients are at the highest risk, while liver and kidney transplant recipients are at lower risk. Of note, most transplant recipients with Nocardia infection were actually on SMX-TMP prophylaxis prior to infection, suggesting that SMX-TMP is not protective against infection. Patients with recent exposure to high-dose glucocorticoids, recent CMV infection, and elevated calcineurin inhibitor levels were at relatively higher risk of infection than matched controls. ${ }^{3.4}$

While this patient had disseminated disease and was symptomatic from her CNS infection, her case represents a relatively uncommon presentation. A majority (77\%) of transplant patients in one large study had only pulmonary disease, whereas only $20 \%$ suffered from disseminated disease. ${ }^{3}$

\section{KEY POINTS}

- A high clinical index of suspicion should be maintained for opportunistic infection in all solid organ transplant recipients, even when their symptomatology is not suggestive of infection.

- Nocardiosis is a difficult to treat infection that can be associated with a wide spectrum of disease but occurs most commonly in the pulmonary form. It is predominantly seen in immunosuppressed populations.

- Disseminated nocardiosis often requires prolonged courses of intravenous antibiotics and multiple source control procedures for adequate treatment.

\section{REFERENCES}

1. Alexander MP, et al. Lesion localization in apractic agraphia. Arch neurol. 1992 42(3):246-51.

2. Wilson JW. Nocardiosis: updates and clinical overview. Mayo Clin Proc. 2012; 87(4):403-7.

3. Peleg AY, et al. Risk factors, clinical characteristics, and outcome of Nocardia infection in organ transplant recipients: a matched case-control study. Clin Infect Dis. 2007; 44(110):1307.

4. Coussement J, et al. Nocardia Infection in Solid Organ Transplant Recipients: A Multicenter European Case-control Study. Clin Infect Dis. 2016; 63(3):338-45 1960;82:183-189. 